\title{
ESTIMATION OF DOSE TO GONADS FROM GAMMA EMITTERS PRESENT IN THE BODY *
}

\author{
W.S. SNYDER, M.R. FORD and G.G. WARNER** \\ (Manuscrit reçu le s juin $\mathrm{1970)}$
}

\begin{abstract}
RÉSUMÉ
L'auteur et ses Collaborateurs ont mis au point un programme du type MonteCarlo pour ordinateur, afin d'estimer la fraction absorbée spécifique $\Phi(X>Y)$, c'est-à-dire l'énergie absorbée par gramme dans $Y$ par rapport à celle dégagée dans $X, X$ étant un organe du corps contenant une source de photons distribuée uniformément et $Y$ un organe-cible. Le programme met en jeu un fantôme mathématique anthropomorphique dont les organes représentent approximativement ceux du prototype quant aux dimensions, à la forme, à la composition et à la densité.
\end{abstract}

The author and colleagues [I] have developed a Monte Carlo type computer code for estimating the specific absorbed fraction of photon energy $\Phi(X-Y)$, i.e., fraction of energy absorbed in $\mathrm{Y}$, per gram, of that released in $X$, where $X$ is an organ of the body containing a source of photons distributed uniformly therein and $Y$ is a target organ. The code involves a mathematical anthropomorphic phantom whose organs approximate those of their prototypes in size, shape, composition, and density. Although results have been published in reference $I$ for 16 source organs $X$ and some 20 target organs $Y$, the estimate is inaccurate sometimes because of poor statistics. This is likely to be the case when $Y$ is a small organ and far from the source. In particular, the estimate of the specific absorbed fraction for the gonads frequently is found to have a large coefficient of variation. For this reason no estimates of the absorbed fraction for gonads were included in reference $\mathrm{I}$ except when the gonads are a source organ. The present note is a first attempt to supply an approximate estimate of the specific absorbed fraction for the gonads.

BERGER [2] has computed the buildup factor $B(\mu x)$ for photons emanating from a point source in an infinite medium of water, giving values for photon energies from 0.02 to $2 \mathrm{MeV}$ and for $\mu x \leqslant 20, \mu$ being the mass attenuation

* Research sponsored by the U.S. Atomic Energy Commission under contract with Union Carbide Corporation. For presentation at Second IRPA Congress, May 3-8, 1970, Brighton, England.

** Health Physics Division - Oak Ridge National Laboratory - Oak Ridge, Tennessee. 
coefficient for the photons. The specific absorbed fraction at distance $x \mathrm{~cm}$ from a point source in the water is then given by :

$$
\Phi(X)=\frac{\mu_{a b} e^{-\mu x} B(\mu x)}{4 \pi x^{2}}
$$

where $x$ represents the distance of the infinitesimal target volume from the point source and $\mu_{a b}$ is the mass absorption coefficient for the photons. If $\Phi(X)$ is used to estimate the fraction of energy absorbed per gram in the gonads at distance $x$ from a point source in the body, one might expect the result be an overestimate since $B(\mu x)$ was computed for an infinite medium so that buildup would be maximized. On the other hand, it is not easy to foresee what effect the differences in composition and density of the body might have in comparing buildup in the body with that in a homogeneous medium. Moreover, even if one suspects ( $\mathrm{I}$ ) will provide an overestimate of the specific absorbed fraction for distances in the body, it is desirable to know approximately the extent of the overestimate.

In an attempt to answer some of the questions raised, $\Phi(X)$ has been computed by the Monte Carlo methods of reference [r] for certain subregions of the body much larger than the gonads so that the statistical accuracy, while not always good, is nevertheless much better than for the gonads. These regions are " slices » shown in Figure $\mathrm{I}$ and numbered $\mathrm{I}, 2,3,4,5$. The source has been placed in the thyroid so that the distance from source to target is as large as can be attained in the trunk. Distance has been taken from a central point of the thyroid to the center of each slice. Clearly distance from the source to a point of the slice varies significantly at different portions of the slice and thus a high degree of accuracy cannot be expected from the comparison. Figures 2-7 show the results for $\Phi(X)$ for photons of energies 2, I, 0.5, 0.2, 0.1, 0.05 MeV as computed by the MonteCarlo calculation of reference [I]. For comparison formula $(\mathrm{I})$ with $B(\mu x)$ as tabulated by Berger is shown also. It is seen that generally formula ( $\mathrm{I}$ ) is higher, as expected, and the cases when this does not hold might plausible be due to either poor statistics or to the rather inaccurate characterization of the distance $x$. To test the influence of this distance factor, the value of $\Phi(x)$ has been computed also for the central portion of the slice (see Fig. I), and it is evident that this generally improves the agreement of the estimate provided by this Monte Carlo calculation and that provided by Berger.

On the basis of the evidence presented here, it seems likely that the use of the buildup factor as given by BERGER [I] and of formula (I) given here will generally overestimate $\Phi(X)$ but not by more than about a factor of 2 . Such a margin of error is acceptable in view of the fact that alternative methods involve uncertainties of at least as much and are frequently much more difficult. It should be noted that the degree of accuracy obtained for formula (I) is roughly the same as can be attained by use of the reciprocity theorem [see Ref. 3], namely, a factor of about 2 .

It should be emphasized that the above comments apply only to the " pointsource " formula. When the source is much more extended, e.g., the lungs or stomach or liver, one should perform an integration over the source region and only then should one expect accuracy as indicated above. The use of a " repre- 
sentative point $)$ for such large organs, say, a central point, will add an additional uncertainty. Moreover, when lungs are the source organ, the distance to the target organ probably should be taken in mass units. The influence of such corrections for the variable density of the body and the choice of " representative points ») for source organs are matters which the author hopes to explore further in future studies.

\section{Acknowledgement}

The author owes much to his colleagues Gordon Warner, who has coded all these calculations for the computer, Mary Rose Ford, who has assisted in designing the code as well as in evaluating and analyzing the data; and James Hickey, who did the sometimes tedious tabulations necessary for this paper and for preparing the figures.

\section{BIBLIOGRAPHIE}

[1] W.S. SNYDER et al., "Estimates of Absorbed Fractions for Monoenergetic Photon Sources Uniformly Distributed in Various Organs of a Heterogeneous Phantom ", MIRD Pamphlet No. 5, Suppl. No. 3, J. Nuclear Med., s (1969).

[2] Martin J. Berger, " Energy Deposition in Water by Photons from Point Isotropic Sources ", MIRD Pamphlet No. 2, Suppl. No. I, J. Nucl. Med., is (1968).

[3] W.S. SNYDER, "Estimation of Absorbed Fraction of Energy from Photon Sources in Body Organs ", Proc. ORAU Symposium on Medical Radionuclides : Radiation Dose and Effects, December 8-11, 1969, Oak Ridge, Tennessee (in press). 


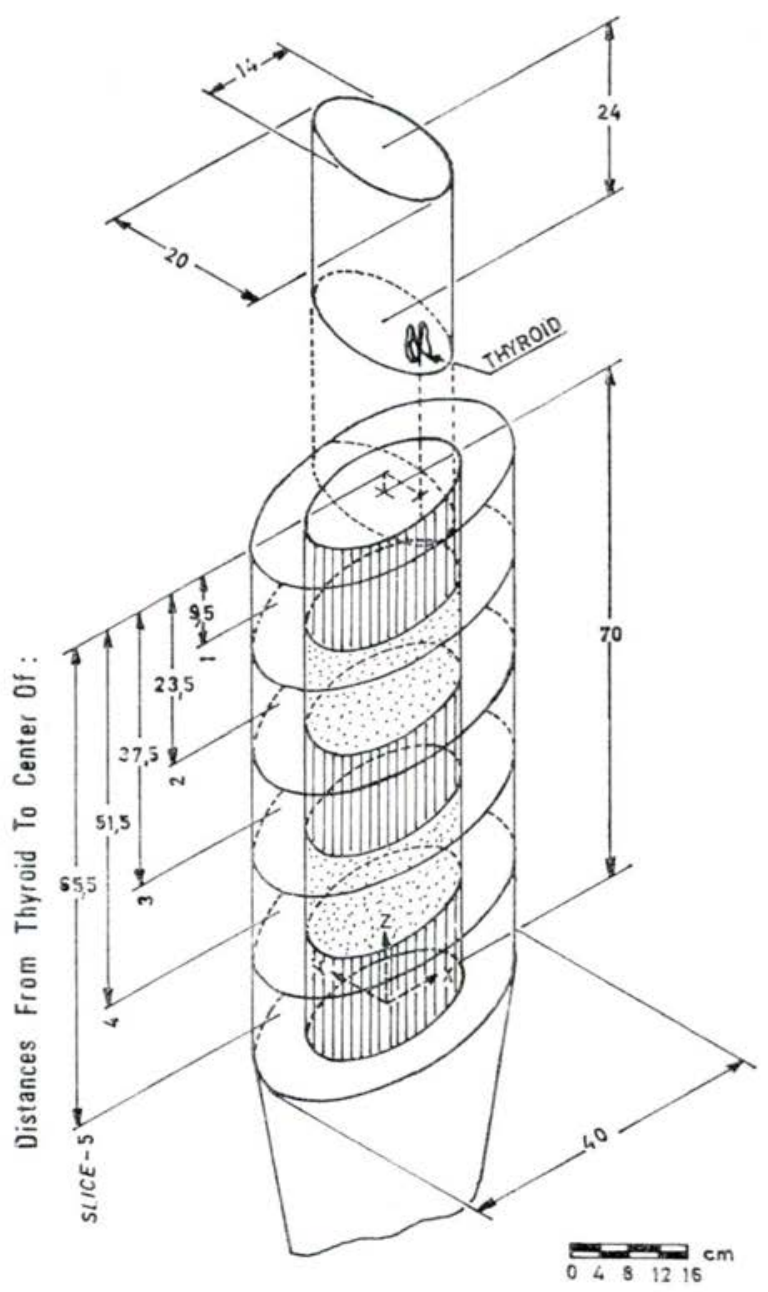

Fig. 1. - The Human Phantom

(Subregions Used for Estimates of Specific Absorbed Fraction) 


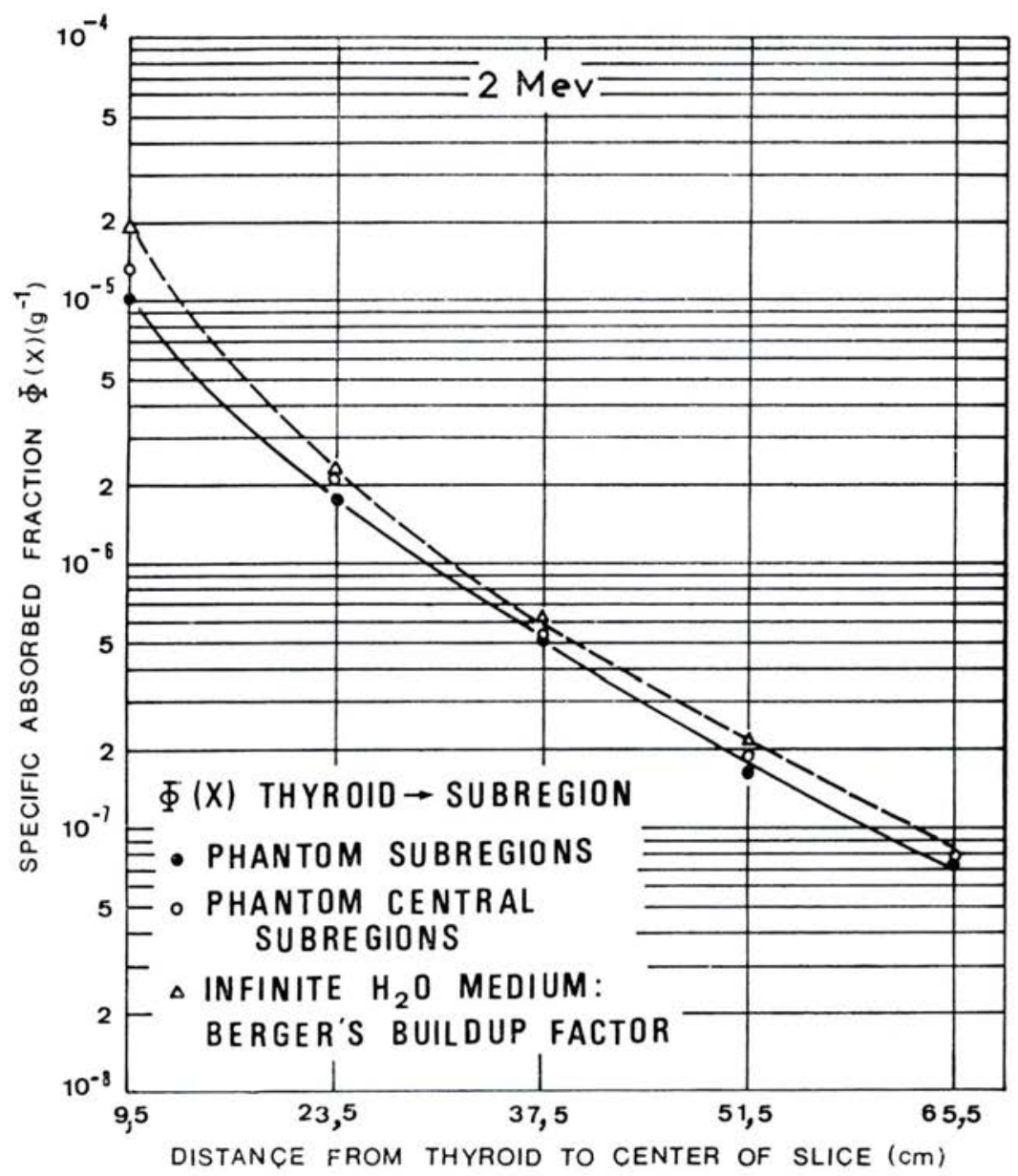

FIG. 2. - Estimates of Specific Absorbed Fraction $\Phi$ for an Infinite Medium of Water and for Subregions of an Anthropomorphic Phantom. 


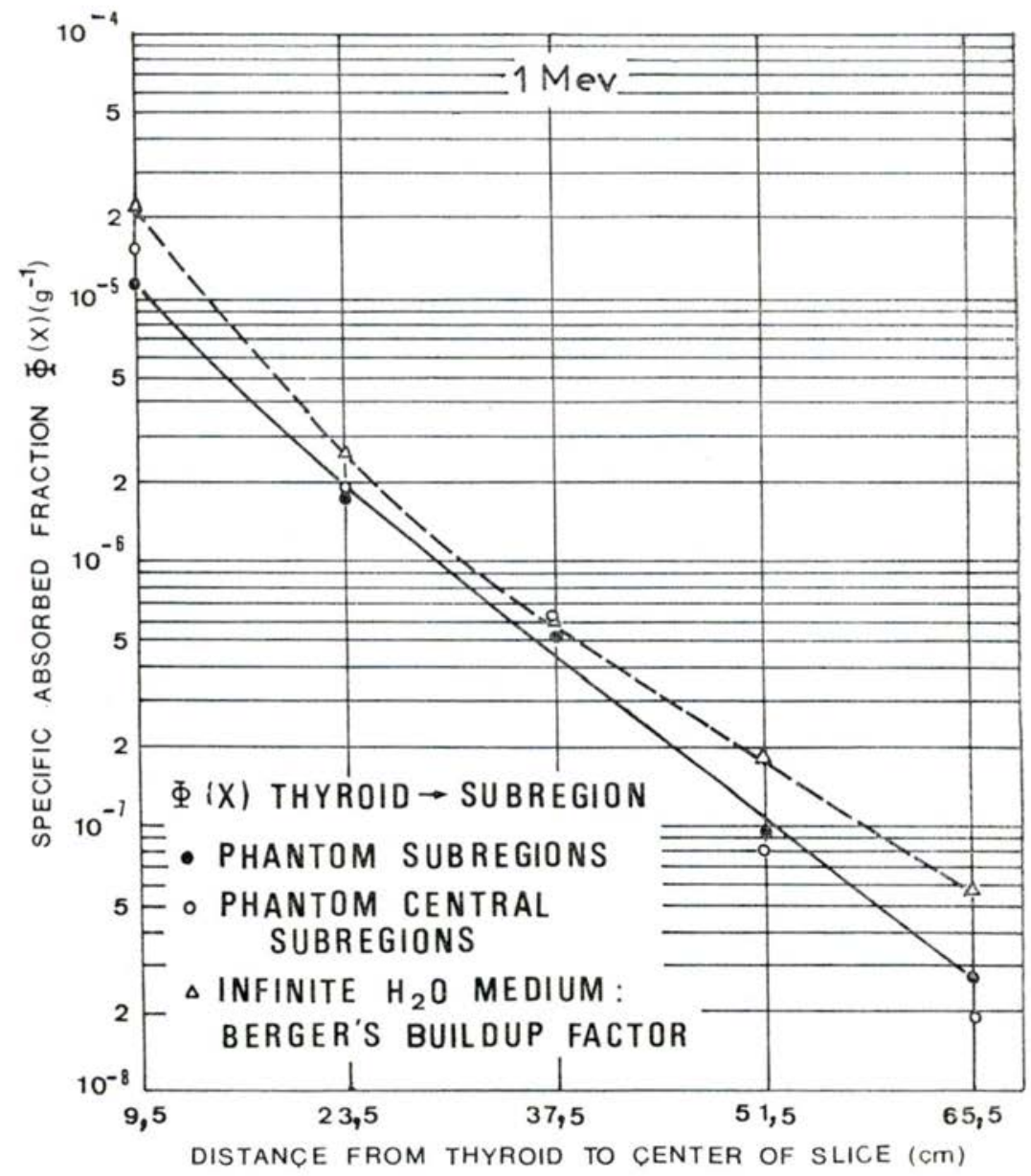

Fig. 3. - Estimates of Specific Absorbed Fraction $\Phi$ for an Infinite Medium of Water and for Subregions of an Anthropomorphic Phantom. 


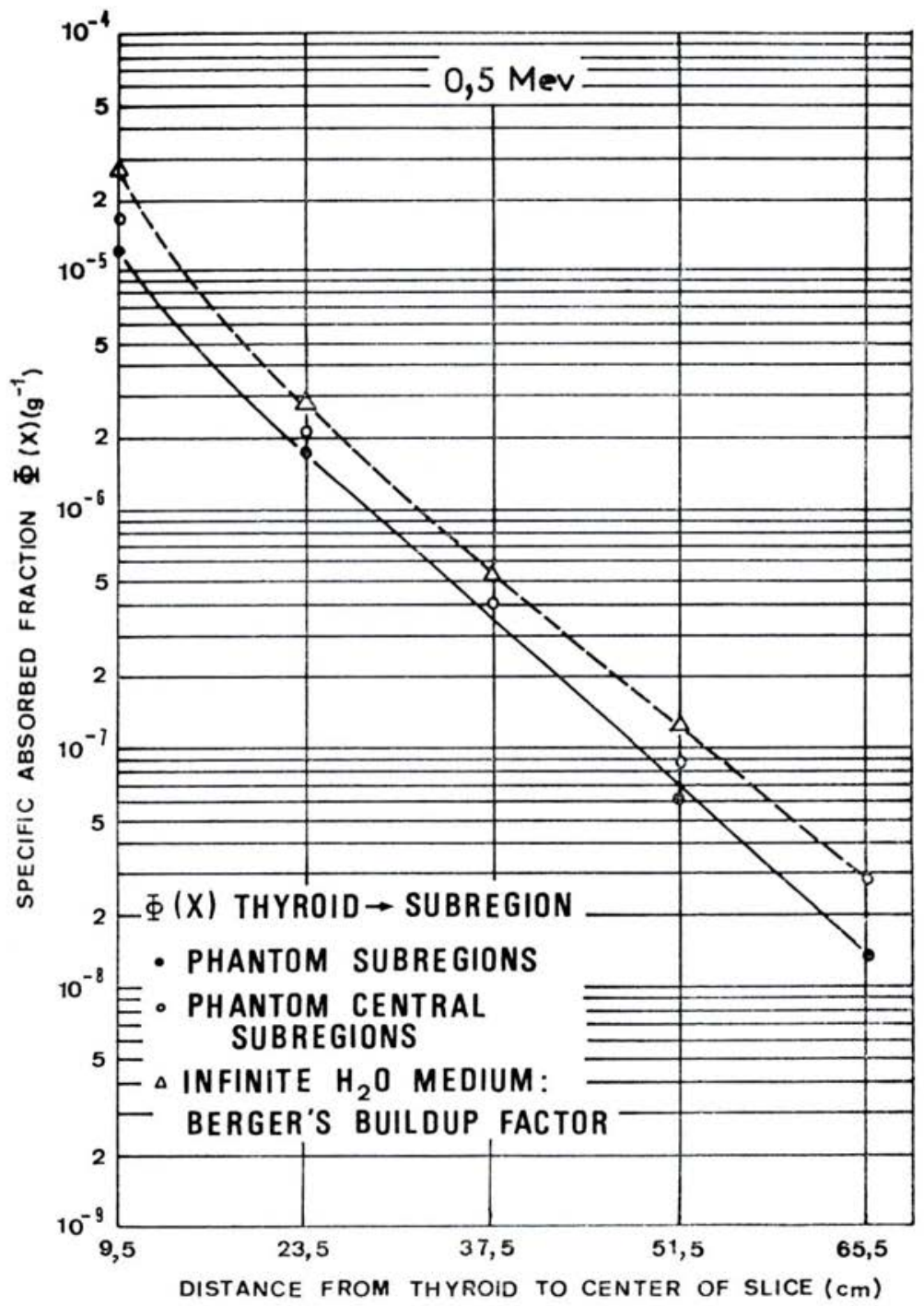

FIG. 4. - Estimates of Specific Absorbed Fraction $\Phi$ for an Infinite Medium of Water and for Subregions of an Anthropomorphic Phantom. 


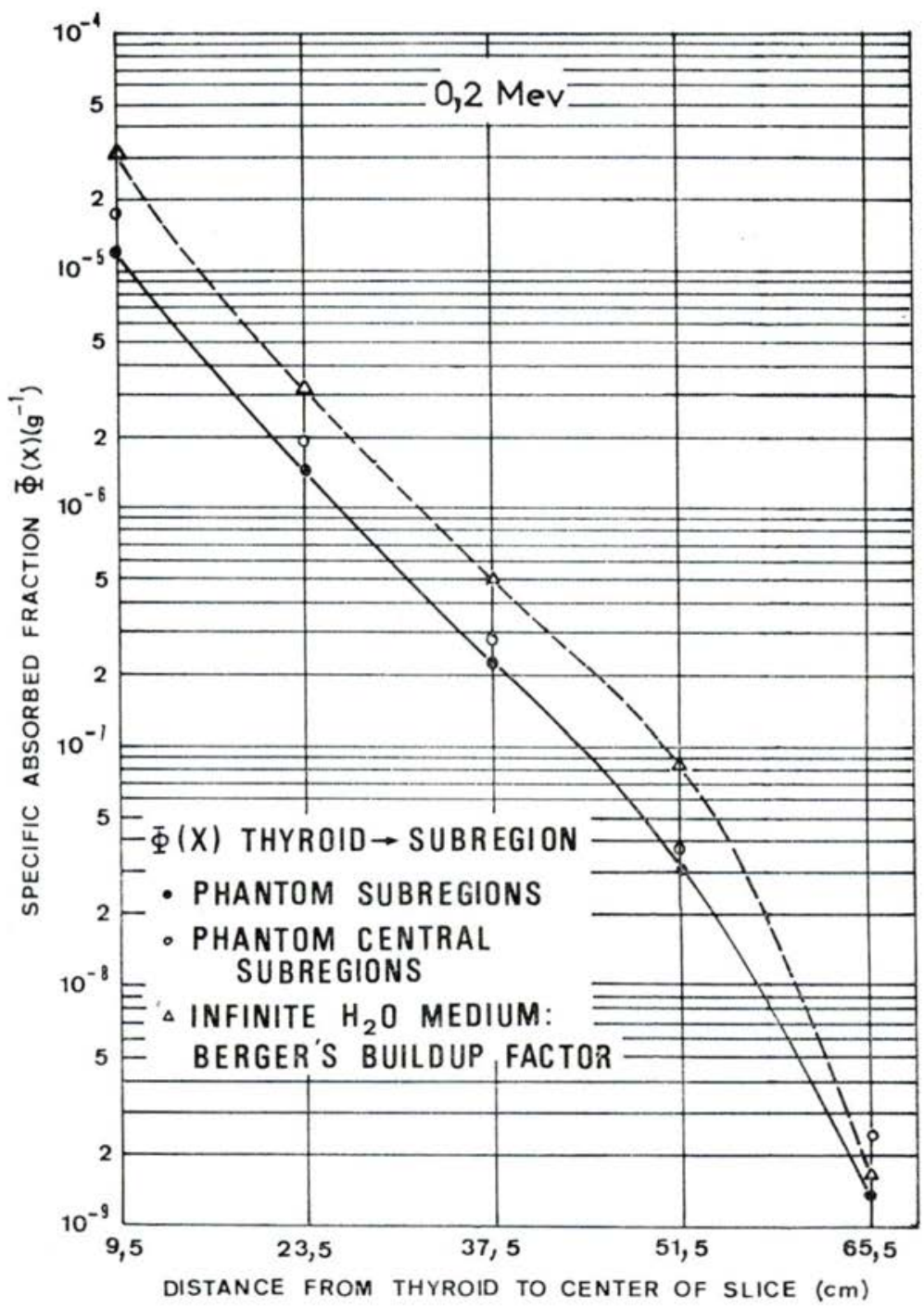

Fig. 5. - Estimates of Specific Absorbed Fraction $\Phi$ for an Infinite Medium of Water and for Subregions of an Anthropomorphic Phantom. 


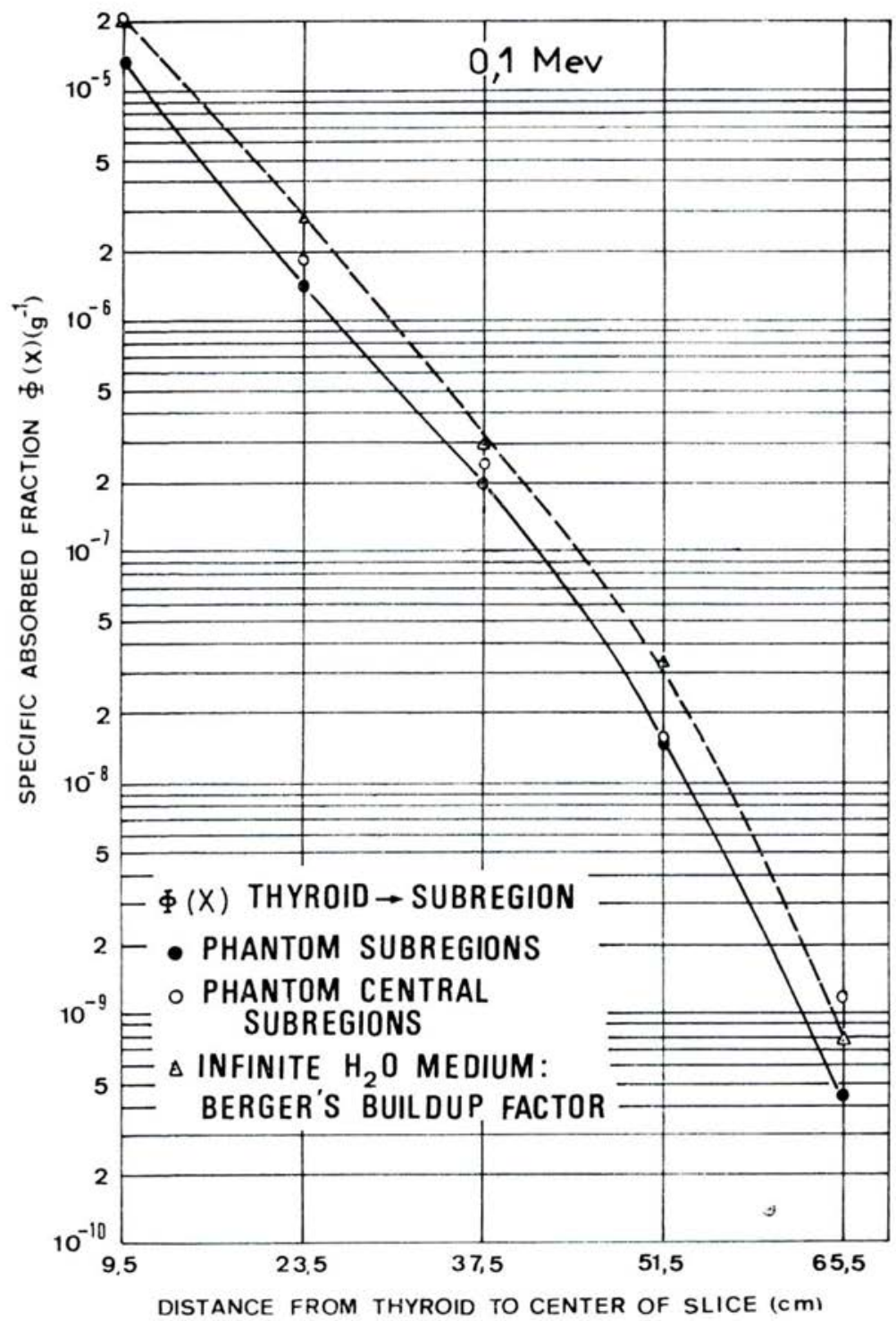

FIG. 6. - Estimates of Specific Absorbed Fraction $\Phi$ for an Infinite Medium of Water and for Subregions of an Anthropomorphic Phantom. 


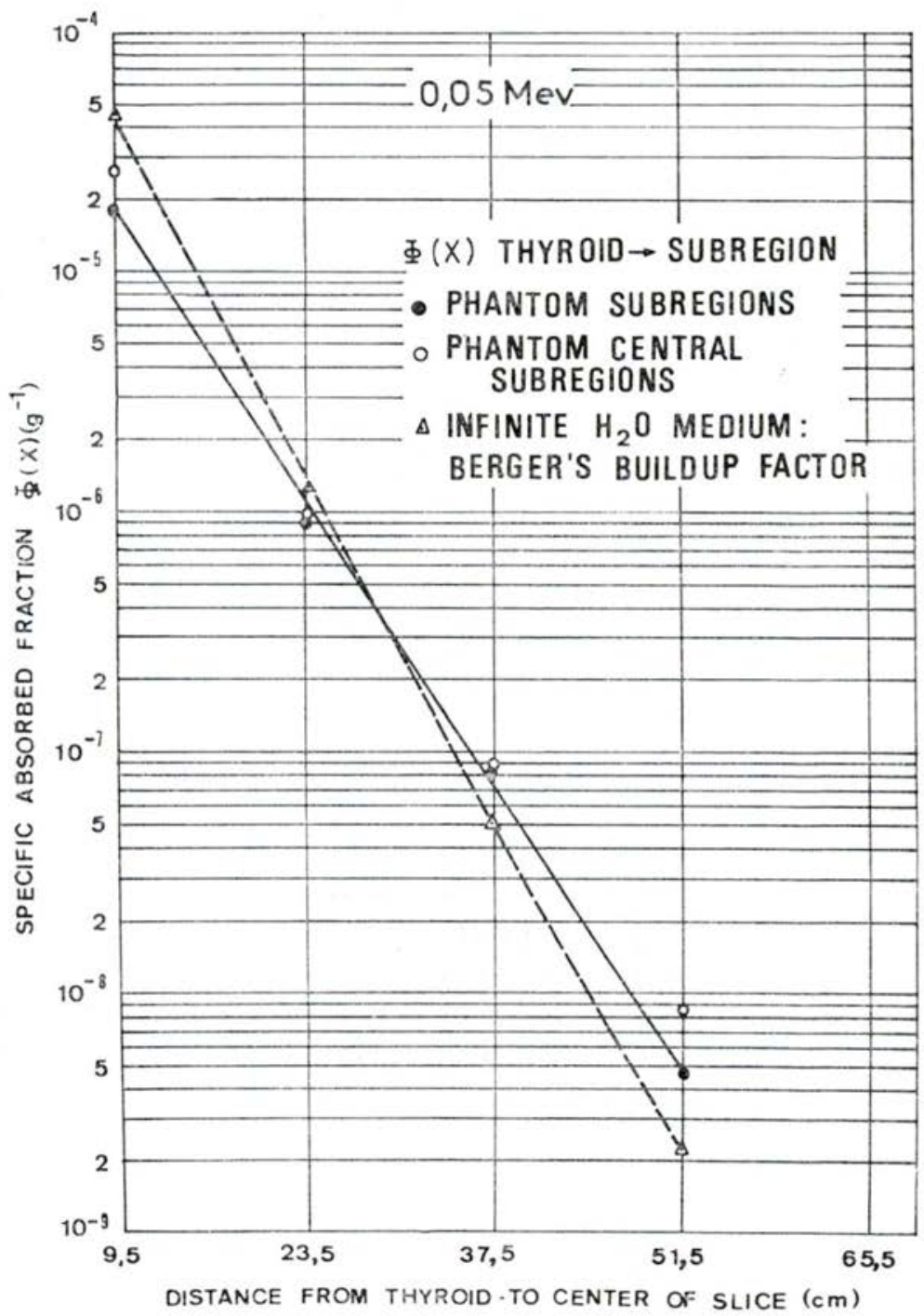

Frg. 7. - Estimates of Specific Absorbed Fraction $\Phi$ for an infinite Medium of Water and for Subregions of an Anthropomorphic Phantom. 